\title{
"INFLUENCIA DEL CLIMA FAMILIAR Y ESTRÉS DEL PADRE DE FAMILIA EN LA SALUD MENTAL DE LOS NIÑOS" *
}

\author{
Alberto Quintana Peña \\ Amparo Sotil Brioso
}

\begin{abstract}
RESUMEN
Investigaciones realizadas en nuestro país señalan la importancia que tiene la familia como factor de riesgo de la aparición de problemas de desarrollo, sociales, interpersonales, educativos) y emocionales en los niños (Benites 1998). En la actual crisis económica del país, en la población de nivel socioeconómico bajo, el padre de familia (o quien haga las veces del mismo), al cumplir la función de ser el proveedor de los recursos económicos necesarios para la supervivencia familiar, es quien experimenta las condiciones estresantes provenientes de la situación de pobreza, desempleo y marginación social en que se encuentran y en consecuencia quien expresa las reacciones emocionales asociadas frente a esta amenaza: ansiedad y cólera. Así, en estas familias surgirían y se reforzarían diversas formas de mensajes violentos vistos y aceptados como hechos normales, (Lamas, 1998). Estas difíciles condiciones de vida afectarían la formación de la personalidad de los niños y adolescentes mermando su desarrollo emocional y social. Pero aún en la población de más escasos recursos se encuentran niños con un desarrollo psicológico adecuado, por ello se rectificó el presente estudio que busca conocer: ¿Que influencia tienen, en la población de extrema pobreza de Lima, el Clima familiar y los acontecimientos estresantes experimentados por el padre de familia en la salud mental de los niños?. Concluyendo que no existe una relación estadísticamente significativa entre el Tipo de Clima familiar y la salud mental de los niños, sin embargo existe una relación significativa entre los acontecimientos estresantes experimentados por el padre de familia y los problemas de salud mental que presentan sus niños.
\end{abstract}

Palabras Clave: Niños. Salud Mental, Clima Familiar: Estrés del Padre de Familia.

\begin{abstract}
Investigations carried out in the Peru point out the importance of the family in the origin of social, interpersonal, educational and emotional problems in the development of the children (Benites 1998). In the current economic crisis of the country, in the population of socioeconomic level under, the family father (or who makes the times of the same one), when completing the function of being the supplier of the necessary economic resources for the family survival, it is who experiences the sressful conditions coming from the situation of poverty, unemployment and social marginalization in that you/they are and in consequence who expressed the associate emotional reactions in front of this threat: anxiety and cholera. This way, in these families they would arise and diverse forms of seen violent messages would be reinforced and accepted as normal facts, (Lamas, 1998). These difficult conditions of life the formation of the personality of the children and adolescents would affect shrinking their emotional and social development. But still in the population of scarcer resources children meet with an appropriate psychological development, for it the present study looks for the know in population is: That it influences do they have the family Climate and the sressful events. experienced by the family father in the mental health of the children?. Concluding that a relationship doesn't exist statistically significant between the Type of family Climate and the mental health of the children and that without place to doubts a significant relationship exists among the sressful conditions experienced by the family father and the problems of mental health that its children present.
\end{abstract}

Key Words: Children. Mental Health. Family Climate. Stress of the Family father.

El autor agradece la participación como miembros de este estudio a los colegas : Dora Frisancho,V., José Davila N., Leoncio Solórzano A., Irma Zarate L. Marina Salazar C. y Jaime Aliaga T. 
El modelo económico que viene implementándose en el Perú ha agudizado los problemas sociales por no responder a las necesidades de desarrollo de la población. Desde el punto de vista de las relaciones humanas, la familia es el centro y núcleo de la sociedad, cuyo papel primordial en el proceso de socialización es el establecimiento de normas, reglas y sobre todo valores éticos y morales. Sin embargo, la actual crisis económica del país distancia a los jefes de familia de esta responsabilidad, ya que ambos tienen que trabajar a fin de satisfacer las necesidades materiales de sus hijos, descuidando un aspecto tan importante como es el factor emocional, el cual es la base de la formación de la personalidad de los niños y adolescentes.

\section{PROBLEMA}

La tasa de población de niños en pobreza extrema cada vez va en aumento (Cuanto, 1997, UNICEF, 1997), llegando a una situación paupérrima. Así en la encuesta de niveles de vida 1985-1986 se informa que el 70\% de los menores de 15 años vivían en hogares con un ingreso promedio mensual de no más de $\$ 15.00$ dólares por persona. A 1990 la pobreza extrema se acentúa y encontramos que son 6'558,800 los niños que sobreviven en condiciones de baja calidad de vida y en situación de riesgo social.

De otro lado, de acuerdo al informe, Panorama Social del INEI de 1996, el 48\% de los niños de 6 a 9 años sufre de desnutrición crónica, siendo esta incidencia mayor en el caso de los niños (54\%) en relación a las niñas (46\%).

En cuanto a la escolaridad se observa que la tercera parte de los alumnos de menores recursos que cursan primaria y secundaria presentan atraso escolar, es decir. tienen edades sistemáticamente mayores a las que les correspondería por grado o año de estudio.

Estas difíciles condiciones de vida a las que están expuestos los niños y adolescentes afectan principalmente la formación de su personalidad creando en ellos normas y reglas erróneas que van a ir poco a poco mermando su desarrollo emocional y social.

Pero aún en la población escasos recursos, en las condiciones de vida más extremas, encontramos niños con un desarrollo psicológico más o menos adecuado (Lasel, 1994), por ello consideramos necesario planteamos la siguiente interrogante: ¿Que influencia tienen, el clima familiar y los acontecimientos estresantes experimentados por el padre de familia en la salud mental de los niños de extrema pobreza de Lima?

\section{SALUD MENTAL INFANTIL}

En un reciente estudio, Livia (1993) estimó la prevalencia de la psicopatología infantil en la población escolar de 6 a 11 años de la zona urbano marginal de ColliqueComas-Lima, en $18 \%$ donde las niñas presentaban mayores transtornos; asociándose como posibles variables condicionantes la desestructuración familiar, bajos niveles educativos y ocupacionales de los padres, lo cual con-firmaría una correlación negativa en nuestro medio entre el nivel socioeconómico y la enfermedad mental, tal como lo señala Feldman (1985).

En la casuística del servicio de psicología del Hospital de Collique (Pimentel 1983) señala como consultas: frecuentes problemas de conducta, bajo rendimiento escolar y problemas de lenguaje. así como características de inseguridad, ansiedad y temor en los niños atendidos.

Boyden (1988) establece en nuestro país que el $46 \%$ de la población infantil de 0-12 años está en riesgo y de este grupo el $15 \%$ en alto riesgo. A ello se agrega la desintegración familiar, hijos no deseados y pobreza extrema. 
Castro (1988) observa que la atención psiquiátrica de los niños en los centros hospitalarios tiene como característica que se pierden el $70 \%$ de pacientes en la quinta consulta y el $90 \%$ en la décima sesión.

Como contra parte, Benites (1998) menciona la existencia de grupos de personas, especialmente niños y adolescentes que a pesar de haber estado, o estar viviendo en situaciones de alto riesgo (pobreza crónica, disponibilidad a drogas, hogares desintegrados, dinámica familiar inadecuada, maltrato infantil, violencia familiar, etc.) han desarrollado ciertos «mecanismos psicológicos» (inmunidad o resiliencia) que los hacen más resistentes a ser afectados por estos factores de riesgo, a involucrarse en conductas antisociales y a desarrollar alteraciones psicológicas de carácter neurótico (ansiedad, fobias, depresión, etc).

\section{FAMILIA EN EL PERÚ}

Una de las funciones primordiales de la familia es la de servir como agente de socialización, de proveer condiciones y experiencias vitales que faciliten el optimo desarrollo biopsicosocial de los hijos.

La mayoría de investigaciones realizadas en nuestro país señalan la importancia que tiene la familia como factor de riesgo o de soporte psicosocial de la aparición en los niños de problemas de desarrollo, sociales, interpersonales, educativos y emocionales. Los estudios sobre estructura familiar, familia desintegrada, contingencias familiares, clima social familiar y crisis familiares así lo demuestran (Benites, 1998).

En nuestro país aún se considera como prototipo de familia adecuada la familia de tipo nuclear, aquella en la que conviven padre, madre e hijos. Sin embargo por condiciones estructurales y sociales (migraciones, desempleo, pobreza, costumbres), se han ido constituyendo otros tipos de familia: extendida, agregada y avuncular. Pareciera ser que la ausencia del elemento paterno o materno (familia avuncular) o la presencia de abuelos y otros familiares (extendida y agregada) pueden influir de diferente manera sobre los hijos dando lugar a patrones de conducta, emociones y pensamientos adaptativos $\mathrm{o}$ desadaptativos según el clima social familiar existente en cada familia en particular.

Benites (1998) encontró que en las familias avunculares los hijos presentaban un menor nivel de autoestima en comparación a sus pares provenientes de familias de tipo nuclear, agregada o extendida concordando con los hallazgos de Bardales (1994). Este autor también halló que la familia agregada proporcionaba mejores condiciones para el desarrollo de la asertividad (sobretodo de la heteroasertividad) que otros tipos de familia.

De otro lado, Espinoza y Col. (1998) en un estudio de 70 familias de nivel socioeconómico bajo, provenientes de siete distritos de Lima, encontraron que los estilos de vida y modelos de interacción familiar inadecuados constituyen factores asociados a problemas de salud mental, siendo mayor cuanto más bajo es el nivel socioeconómico, la familia es más antigua y sobre todo si es extendida.

Pimentel (1988) ha descrito en las zonas urbano marginales la violencia familiar entendiendo por ella las acciones realizadas por medio de la fuerza por uno o más miembros de la familia sobre uno o más de la misma; Sea para imponer sobre ellos la realización de algún acto en contra de su voluntad, sea para castigarles por algún acto cometido o dejado de hacer, concluyendo que en este medio la estructura autoritaria y la ideología del castigo físico y verbal son una forma de relación cotidiana entre padres e hijos. Coincidiendo con Majluf (1989) quien encontró que las madres de los niveles socio económicos más bajos son autoritarias, restrictivas y punitivas.

Lamas (1998) afirma que en nuestro país, para muchas familias poder hacerle frente a la vida (es decir, a la pobreza, al desempleo, a la marginación social) exige dos esfuerzos de naturaleza bien distinta: sobrevivir y salir de la pobreza. A estas dos exigencias se subordinarían todas las demás actividades, necesidades y demandas de la familia, las cuales 
tendrían como interferente principal al niño. Concluyendo que en la familia surgirían y se reforzarían diversas formas de violencia que trastocarían su integridad y sobre todo rebasarían su capacidad de control efectivo viendo invadido su interior por una imposición de mensajes violentos que una vez internalizados serian vistos y aceptados como hechos normales.

\section{ESTRESORES Y REACCIONES EMOCIONALES DEL ESTRÉS}

Spielberger y Moscoso (1996) citando a Lazarus argumentan que el estrés no puede ser definido en términos de estímulos o respuesta. sino más bien como variable interviniente. Añadiendo que las transacciones de estrés pueden ser iniciadas por cualquier estímulo o situación que es percibida como potencialmente peligrosa, dañina o frustrante (estresor). Definiendo el termino estresor como una situación de estimulo o evento que es objetivamente caracterizado por un cierto grado de peligro físico o psicológico, precisando sin embargo que situaciones objetivamente benignas pueden también ser percibidas como peligrosas por ciertos individuos.

En el momento que una situación es percibida como amenaza, al margen de que el peligro sea real o imaginario, el sentido de amenaza va a generar una reacción emocional desagradable. En consecuencia, estrés podría ser definido como un proceso que incluye transacciones entre el individuo y su medio ambiente durante el cual los estresores están íntimamente conectados con reacciones de ansiedad y cólera a través de la percepción de amenaza.

Así la ansiedad y la cólera resultan ser reacciones emocionales primarias, producto de la evaluación cognitiva de amenaza. Un estado de ansiedad consistiría en sentimientos de nerviosismo, preocupación, tensión y aprehensión, e incluiría la excitación del sistema nervioso autónomo. Este estado emocional puede variar desde una ligera aprehensión hasta un temor intenso o pánico. De igual forma un estado de cólera consistiría de sentimientos que pueden variar desde una ligera irritación o fastidio hasta una rabia intensa y furia, con un excitación del sistema nervioso autónomo equivalente a la intensidad de estos estados de cólera.

En la población de nivel socioeconómico bajo el padre de familia (o quien haga las veces del mismo), al cumplir la función de ser el proveedor de los recursos económicos que permiten a la familia la adquisición de los bienes y servicios necesarios para su supervivencia, es quien más probablemente experimente las condición estresantes provenientes de la situación de pobreza, desempleo y marginación social en que se encuentran $y$ en consecuencia quien más probablemente exprese las reacciones emocionales asociadas.

El presente estudio tiene como propósito el:

- Conocer en los pobladores del nivel socioeconómico bajo de Lima las variables intrafamiliares que determinan la Salud Mental de los niños.

- Conocer el grado en que los diversos tipos de clima familiar existentes afectan la salud mental de los niños.

- Conocer el grado en que los acontecimientos estresantes experimentados por el padre de familia (o quien haga las veces del mismo) afectan la salud mental de los niños. 


\section{VARIABLES}

Variable Dependiente: Salud mental de los niños.

Variable independiente 1 (no manipulada): Clima social familiar.

Variable independiente 2 (no manipulada): Acontecimientos estresantes experimentados por el padre de familia.

Variables controladas:

- Área geográfica, Lima metropolitana.

- Nivel socioeconómico bajo

\section{HIPOTESIS}

\section{General:}

- Existe una relación significativa entre el número de síntomas de problemas de salud mental que presentan un grupo de niños de nivel socioeconómico bajo y las variables tipo de clima familiar y número de situaciones estresantes a que esta expuesto el padre de familia (o quien haga las veces del mismo).

\section{Especificas}

- Existe una relación significativa entre el número de situaciones estresantes a que esta expuesto el padre de familia (o quien haga las veces del mismo) y el número de síntomas de problemas de salud mental que presentan sus niños.

- Los niños de familias del tipo Extremo en el modelo circunflejo de Olson, presentan significativamente más problemas de salud mental que los niños de familias del tipo Balanceado en este mismo modelo.

\section{METODO}

Este estudio es de carácter ex postfacto. Su diseño es el de Grupo de criterio.

\section{Sujetos}

La población a estudiar estará conformada por familias Limeñas del nivel socioeconómico bajo. Específicamente de Barrios altos por ser ésta una de las zonas del cercado de Lima donde la crisis socioeconómica que experimentamos, con el consiguiente deterioro en los niveles de salud y educación ha impactado con mayor severidad. Haciendo característico de esta área el hacinamiento de familias en casonas convertidas en grandes tugurios con deficientes condiciones de salubridad, así como problemas sociales del tipo delincuencia, alcoholismo, drogadicción y desempleo entre otros. La técnica elegida para seleccionar la muestra es el muestreo no probabilístico de tipo intencional. Se estudió a 75 familias, muchas de ellas con más de un niño, por lo que la muestra de niños estuvo constituida por 92.

\section{INSTRUMENTOS}

Ficha demográfica: Permite registrar algunas características relevantes de las familias estudiadas: Edad, sexo, grado de instrucción, etc. 
Cuestionario Faces II: Desarrollado por Olson, Sprenkle y Russell, con el objetivo de obtener una perspectiva del interior del funcionamiento familiar tal como es percibido por diversos miembros de la familia. El cual esta conformado por una escala de evaluación de la adaptabilidad y otra de la cohesión Familiar. Traducido y validado en Lima por Malamut, quien halla un índice de correlación de Pearson entre las mitades de 0.58 para cohesión y de 0.54 para adaptabilidad. Además del índice de consistencia interna de Spearman-Brown, donde se obtuvo 0.73 para cohesión y de 0.70 para adaptabilidad. Por otro lado se encontró un coeficiente alfa de Cronbach de 0.74 para cohesión y de 0.63 para adaptabilidad. Esta prueba evalúa los diferentes tipos de sistemas familiares de acuerdo al Modelo Circumplejo de David Olson el cual a partir de la combinación de cuatro niveles de Cohesión con cuatro niveles de adaptabilidad familiar permite identificar dieciséis tipos de sistemas maritales y tres tipos de familias, a saber: Balanceada, Rango medio y Extrema. Se aplica a partir de los 12 años.

La Adaptabilidad familiar examina el grado en que el sistema familiar es flexible y capaz de cambiar. Se define como la habilidad de un sistema marital o familiar para cambiar su estructura de poder, las relaciones de roles y las reglas que rigen las relaciones, en respuesta al estrés situacional y propio del desarrollo. Los conceptos específicos para medir y diagnosticar esta dimensión son: autoridad dentro de la familia (1iderazgo, asertividad, control y disciplina), estilos de negociación, relaciones entre roles y reglas que rigen las relaciones.

Los grados de adaptabilidad oscilan entre rígida, estructurada, flexible y caótica:

Rígida; se caracteriza por el liderazgo autoritario y autocrático, disciplina estricta, rígida y de aplicación severa, los padres imponen decisiones, las reglas se hacen cumplir estrictamente no habiendo posibilidad de cambio y con roles estrictamente definidos.

Estructurada; se caracteriza por ser democrática, los padres toman decisiones, siendo la disciplina rara vez severa y predecibles sus consecuencias, los roles son estables pero pueden compartirse, las reglas se hacen cumplir firmemente y pocas son las que cambian, el principio del liderazgo es autoritario, siendo algunas veces igualitario.

Flexible; se caracteriza por un liderazgo igualitario y permitir cambios, la disciplina rara vez es severa y sus consecuencias son predecibles, usualmente es democrática y hay acuerdo en las decisiones, se comparten los roles y las reglas se hacen cumplir con flexibilidad y algunas reglas cambian.

Caótica; se caracteriza por un liderazgo limitado e ineficaz, disciplina poco severa e inconsistente, falta de claridad en los roles, decisiones parentales impulsivas y frecuentes cambios en las reglas que se hacen cumplir inconsistentemente.

El Modelo Circumplejo postula que los niveles de adaptabilidad estructurada y flexible facilitan el funcionamiento conyugal y familiar, mientras que los extremos. rígida y caótica, resultan problemáticos para las familias.

La Cohesión familiar examina el grado en que los miembros de la familia están compenetrados y se ayudan y apoyan entre sí. Se define como el vínculo emocional que los miembros de la familia mantienen entre ellos. Los conceptos específicos para medir y diagnosticar esta dimensión son: vinculación emocional, dependencia, límites, coaliciones parento-filiales, tiempo, espacio, amigos, toma de decisiones, intereses y recreación.

Los grados de cohesión oscilan entre desligada, separada, conectada y aglutinada:

Desligada; se caracteriza por la extrema separación emocional entre sus miembros, poca interacción, falta de cercanía parento filial, con predominio de la separación personal preferencia de espacios separados, intereses desiguales focalizados fuera de la familia. 
Separada; se caracteriza por la separación emocional pero en ocasiones se demuestra la correspondencia afectiva, aceptar el involucramiento pero preferir la distancia, límites parento filiales claros, se considera importante el tiempo individual pero pasan parte del tiempo juntos, se prefieren los espacios separados pero comparten el espacio familiar, las decisiones se toman individualmente pero hay posibilidad de decisiones conjuntas, interés focalizado fuera de la familia. la recreación se lleva a cabo más separada que compartida.

Conectada; se caracteriza por la cercanía emocional, las interacciones élfectivas son alentadas y preferidas, la lealtad familiar es esperada, el interés se focaliza dentro de la familia, enfatiza la interrelación pero se permite la distancia personal, la necesidad separación es respetada pero no valorada. los límites entre los subsistemas son claros con cercanía parento filial. los amigos individuales y preferencias se comparten.

Aglutinada: se caracteriza por el involucramiento simbiótico, dependencia entre sus miembros, expresada con alta reactividad emocional, permiten poco tiempo y espacio privado, interés focalizado en la familia, hay coaliciones parento filiales.

El Modelo Circumplejo postula que los niveles de cohesión separada y conectada, facilitan el funcionamiento familiar, mientras que los extremos, desligada y aglutinada, resultan problemáticos para las familias.

El tipo de familia Balanceado de acuerdo al Modelo Circumplejo es el más adecuado y corresponde a las familias resultantes de la combinación de los siguientes grados de adaptabilidad y cohesión: flexiblemente separada, flexiblemente conectada, estructural mente separada y estructural mente conectada; FS, FC, ES y EC, respectivamente.

El tipo de familia Rango Medio corresponde a las familias resultantes de la combinación de los siguientes grados de adaptabilidad y cohesión: caóticamente separada, caóticamente conectada, flexiblemente desligada, flexiblemente aglutinada, estructuralmente desligada, estructuralmente aglutinada, rígidamente separada y rígidamente conectada; CS, CC, FO, FA, E.O, EA, RS y RC, respectivamente.

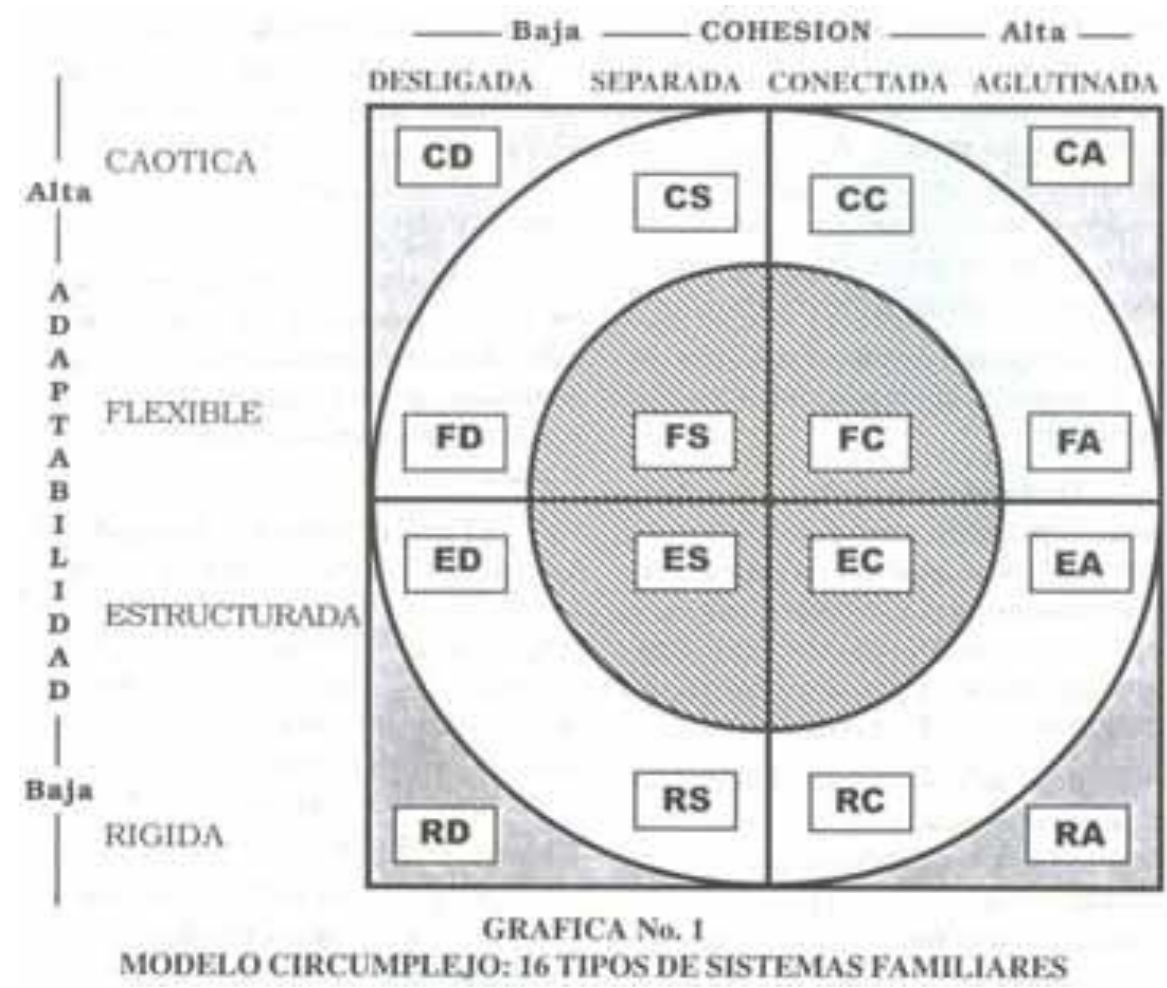


El tipo de familia Extremo de acuerdo al Modelo Circumplejo es el menos adecuado y corresponde a las familias resultantes de la combinación de los siguientes grados de adaptabilidad y cohesión: caóticamente desligada, caóticamente aglutinada, rígidamente desligada y rígidamente aglutinada. CO. CA. RO y RA. respectivamente.

La escala de acontecimientos estresantes: Que es una lista de chequeo derivada del enfoque conductual cognitivo del estrés con 52 posibles situaciones estresantes la cual permite chequear la ocurrencia o no de un acontecimiento y la intensidad de su impacto emocional en unidades subjetivas de disturbio (SUD). Se consideró como criterio declarar haber sentido un grado de tensión mayor o igual a 06 en una escala de unidades subjetivas de disturbio $\mathrm{O}$ a 10, y que dicho acontecimiento se haya llevado a cabo en el último año.

Cuestionario de Síntomas para Niños (RQC): Lista de chequeo derivada de la psiquiatría infantil y recomendada por la Organización Mundial de la Salud que permite una rápida y panorámica exploración del estado de salud mental del niño a través 10 reactivos dicotómicos de calificación $1 / 0$

\section{PROCEDIMIENTO DE RECOLECCIÓN DE DATOS}

- Establecer coordinaciones con las organizaciones de base de los solares (casonas), a fin de acceder a las unidades de estudio.

- Recoger la información, administrando los instrumentos casa por casa.

- Hacer el depurado y consolidado de los datos en RQC.

- Analizar en relación a los síntomas de déficit de salud mental de los niños, los tipos de clima social familiar y el número de situaciones estresantes experimentado por el padre de familia que les corresponde. y someterlos al procesamiento estadístico.

\section{ANÁLISIS ESTADÍSTICO}

Análisis de la correlación existente entre el déficit de salud mental de los niños y las variables estudiadas.

Estimar en que medida las variables Tipo de clima familiar y estrés del padre permiten predecir la salud mental del niño, por medio de una regresión lineal.

\section{RESULTADOS}

En la Tabla No. 1 se observan las Normas y los puntos de corte en las familias de la muestra para los cuatro niveles de Adaptabilidad y Cohesión, apreciándose que en Adaptabilidad los puntajes estuvieron entre 13 y 40, con un promedio de 29.4 y una desviación standard de 7.3. En Cohesión los puntajes fluctuaron entre 0 y 28, con un promedio de 16.2 y una desviación standard de 6.7 . 
Tabla No. 1

Normas y puntos de corte

$(n=75)$

\begin{tabular}{|l|c|c|}
\hline \multirow{2}{*}{ Adaptabilidad } & Promedio & D.S. \\
\cline { 2 - 3 } Cohesión & 29.4 & 7.3 \\
\hline Adaptabilidad & 16.2 & 6.7 \\
\cline { 2 - 3 } Rntervalo & \% \\
\cline { 2 - 3 } Estructurada & $13-22$ & $17.3 \%$ \\
Flexible & $23-29$ & $46.7 \%$ \\
Caótica & $30-36$ & $25.4 \%$ \\
Cohesión & & $10.6 \%$ \\
Desligada & $0-9$ & 10.7 \\
Separada & $10-16$ & 37.3 \\
Conectada & $17-23$ & 37.3 \\
Aglutinada & $24-28$ & 14.7 \\
\hline
\end{tabular}

Como se observa en la Tabla No. 1, las familias de la muestra se distribuyeron en los distintos niveles de adaptabilidad y cohesión de la siguiente forma:

En adaptabilidad se encuentran; familias rígidas entre 13 y 22 (17.3\%), estruc-turadas entre 23 y 29 (46.7\%), flexibles entre 30 y 36 (25.4\%), Y caóticas entre 37 y 40 (10.6\%). Destacando nítidamente el mayor porcentaje de las familias estructuradas.

En cohesión se encuentran; familias desligadas entre 0 y 9 (10.7\%), separadas entre 10 y $16(37.3 \%)$, conectadas entre 17 y 23 (37.3\%), y aglutinadas entre 24 y $28(14.7 \%)$. Destacando las familias con niveles de cohesión que facilitan el funcionamiento familiar e incluyen al 74.6\% (2/3) de la muestra.

La distribución porcentual de las familias de la muestra en cada uno de los 16 tipos de familias del modelo circumplejo, lo observamos en la Gráfica No. 2.

Como puede observarse en la Gráfica No. 2, a pesar de las condiciones de pobreza en que viven las familias del estudio, predominan entre ellas los tipos de familias Balanceadas (56.0\%), siendo bastante pequeño el porcentaje de familias inadecuadas, es decir, extremas (apenas el 9.3\%).

En la tabla 2 pueden observarse los resultados de la lista de chequeo de los 52 acontecimientos estresantes (ver anexo), agrupados en siete categorías por su afinidad temática. En ella se puede apreciar que el mayor porcentaje de situaciones estresantes que experimentan los jefes de familia de la muestra se encuentran en la categoría de pobreza, familia e hijos que juntas abarcan el $60.2 \%$ del total, así mismo en la Gráfica No. 3 se puede apreciar que aproximadamente un tercio de los padres $(31.0 \%)$, no son estresados por más de una situación. 


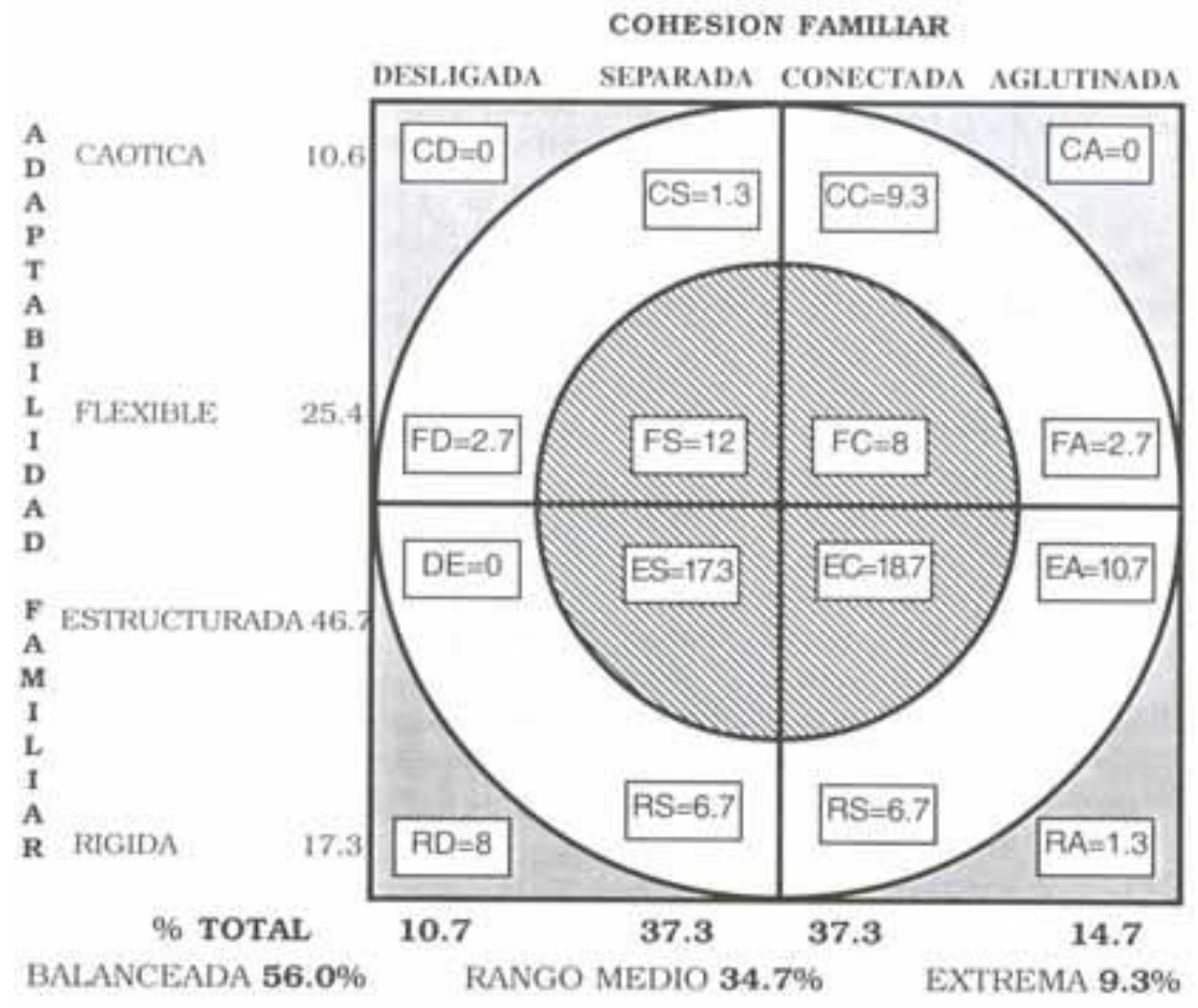

TABLA No. 2

Porcentaje de Jefes de Familia que experimentan determinada categoria de acontecimientos estresantes

\begin{tabular}{|l|l|r|c|}
\hline Categaria & \multicolumn{1}{|c|}{ Items } & $N^{\circ}$ Items & \multicolumn{1}{|c|}{$\%$} \\
\hline Parcja & $1,2,3,4,5,6,7,8,9,10,11,52$ & 12 & $11.8 \%$ \\
Hijos & $12,13,21,22,23,41,42$ & 7 & $16,4 \%$ \\
Familia & $14,15,16,17,18,20,38,39,46,47,48,49,50$ & 13 & $23,7 \%$ \\
Pobreza & $28,29,43,44,45$ & 5 & $20.1 \%$ \\
Vecindad & $19,32,33,40$ & 4 & $8.9 \%$ \\
Trabajo & $24-27$ & 4 & $7.9 \%$ \\
Otros & $30,31,34,35,36,37,51$ & 7 & $11.2 \%$ \\
\hline TOTAL & & 52 & $100 \%$ \\
\hline
\end{tabular}




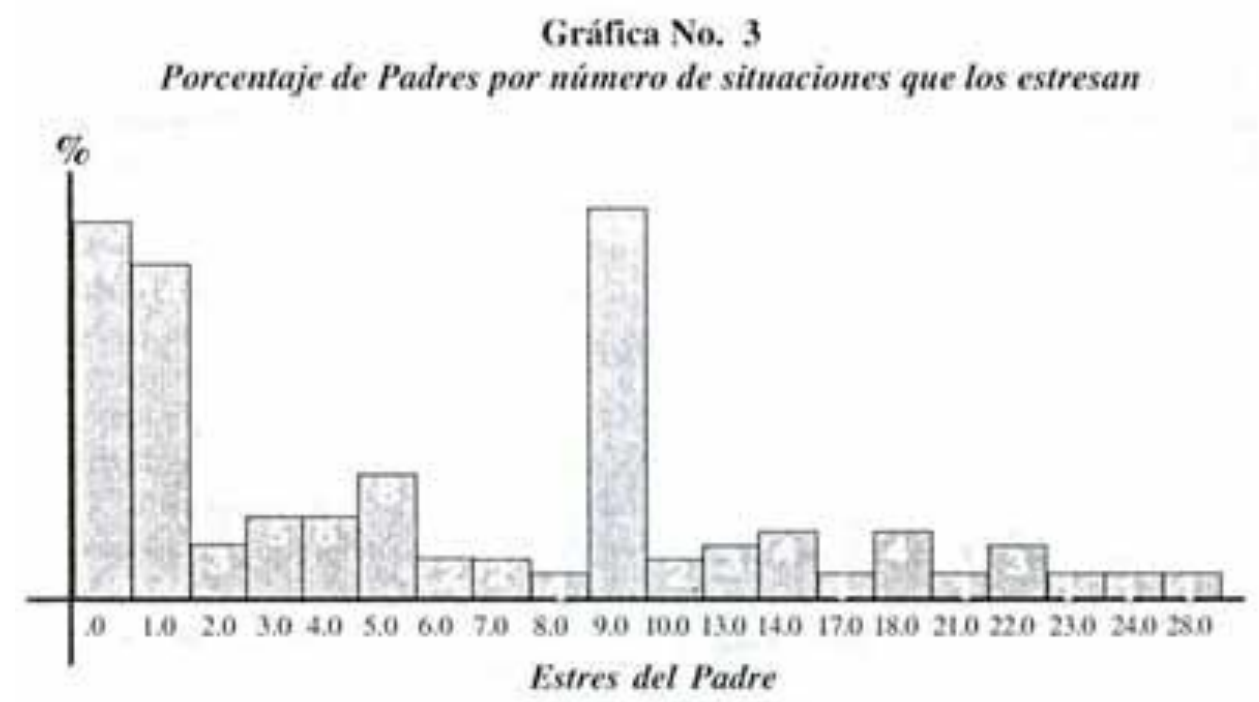

En la Gráfica No. 4 consignamos el porcentaje de niños que experimento determinada cantidad de síntomas, resaltando el hecho que la mayoría de los niños $62.6 \%$, no presento más de un síntoma. A su vez en la tabla 3 se observa el porcentaje de niños (de un total de 92), que presento cada uno de los diez síntomas del cuestionario de síntomas de salud mental.

\section{Gráfica No. 4}

Porcentaje de niños por cantidad de sintomas

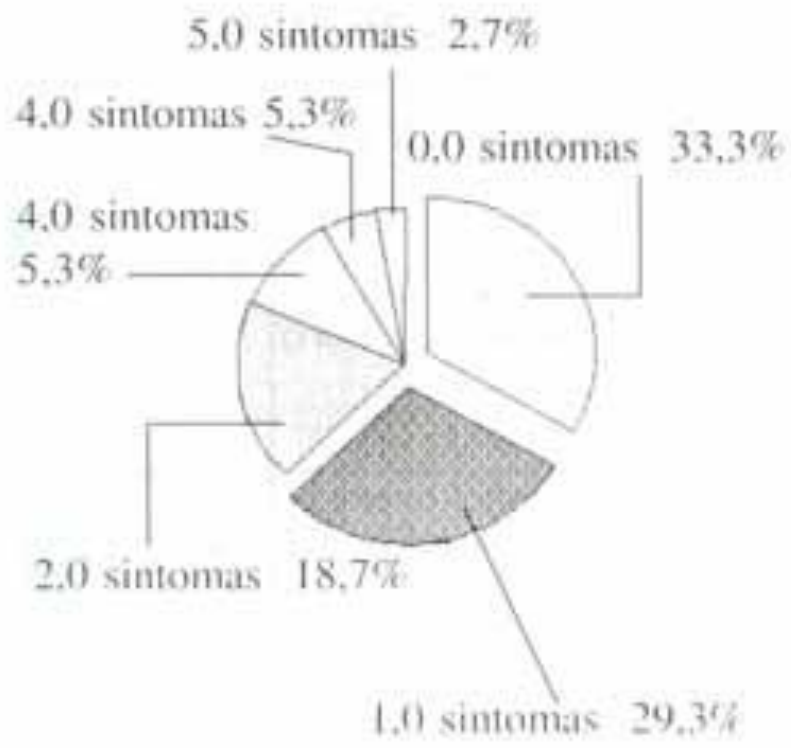


Tabla No. 3

Porcentaje de niños por cada uno de los sintomas

\begin{tabular}{|c|}
\hline SINTOMA \\
\hline Lenguaje anormal en alguna forma ...... 22.1 \\
\hline Dormirmal 9.5 \\
\hline $\begin{array}{l}\text { Tener ocasionalmente } \\
\text { convulsiones o desvanecimientos ...... } 10.5\end{array}$ \\
\hline $\begin{array}{l}\text { Sufrir de frecuentes dolores } \\
\text { de cabeza }\end{array}$ \\
\hline Huir de casa frecuentemente ................. 2.1 \\
\hline Robar cosas de la casa .................. \\
\hline $\begin{array}{l}\text { Asustarse o ponerse nervioso } \\
\text { sin razón ...................................... } 20.0\end{array}$ \\
\hline $\begin{array}{l}\text { Parecer retardado o lento } \\
\text { para aprender }\end{array}$ \\
\hline Casi nunca jugar con otros niños ....... 16.0 \\
\hline Orinarse o defecar en su ropa ............ 19.0 \\
\hline
\end{tabular}

En esta tabla se aprecia que destacan los síntomas: Lenguaje anormal en alguna forma. Asustarse o ponerse nervioso sin razón. Orinarse o defecar en su ropa y Casi nunca jugar con otros niños. como los mas frecuentemente presentados por los niños del estudio.

Los niños de la muestra fueron mayormente del sexo femenino (61.3\%, ver Fig. 5), en su mayoría no asisten a una escuela (62.7\%, ver Fig 6), y sus edades oscilaban entre uno y doce años siendo las edades mas frecuentes tres años (22.7\%) y seis años (14.7\% ver Fig 7)

\section{Gráfica No. 5}

Genero de los niños

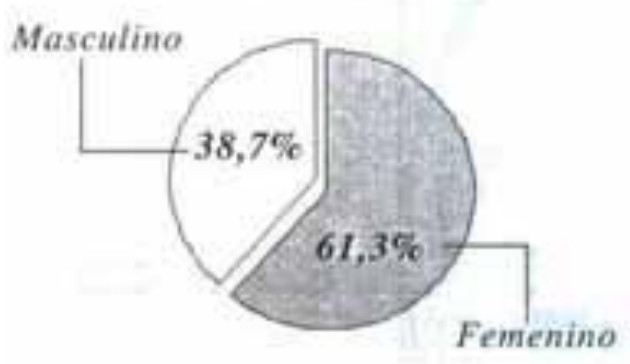

Gráfica No. 6

Escolaridad de los niños

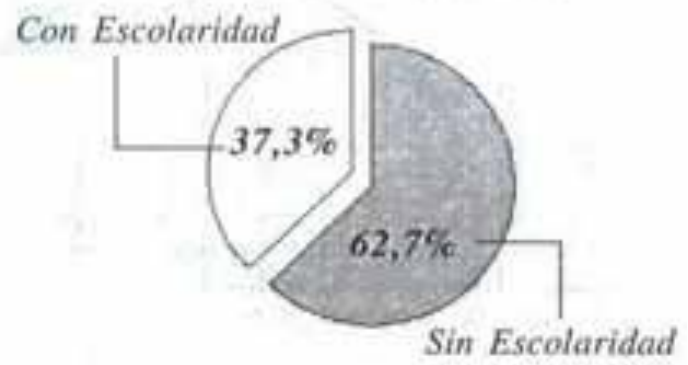




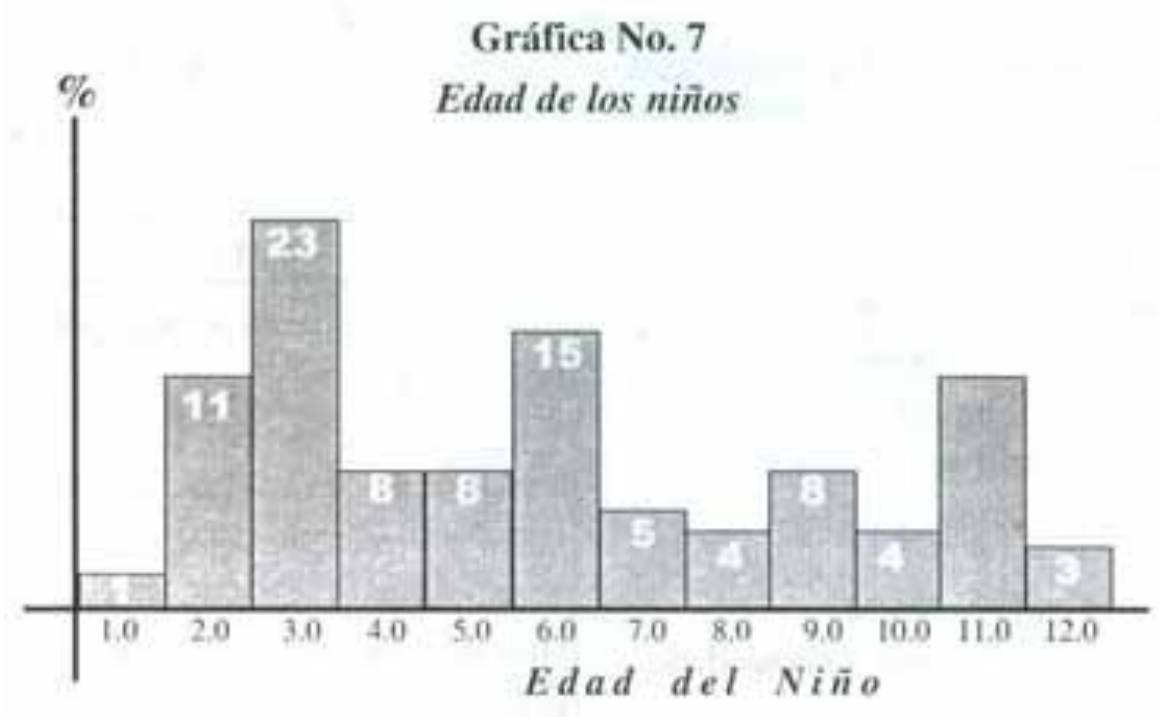

Los datos de la tabla No. 4 muestran los hallazgos obtenidos al contrastar la primera hipótesis especifica, en ellos puede observarse el coeficiente de con-elación de Pearson de 0.332 significativo en el nivel de 0.01 , el cual nos indica que a mayor numero de acontecimientos estresantes experimentados por el padre de familia (o quien haga veces del mismo). tiende a haber un mayor índice de deterioro de la salud mental de sus niños.

Tabla $N^{\circ} 4$

Correlación entre el estrés del padre y la salud mental de los niños

\begin{tabular}{|c|c|c|}
\hline & SM & $\begin{array}{c}\text { Padres } \\
\text { TR }\end{array}$ \\
\hline $\begin{array}{l}\text { SM } \\
\text { Pearson Correlatión } \\
\text { Sig. } \\
\text { (2-tailed) } \\
\end{array}$ & $\begin{array}{l}1.000 \\
92\end{array}$ & $\begin{array}{l}.332 * * \\
.001 \\
92\end{array}$ \\
\hline 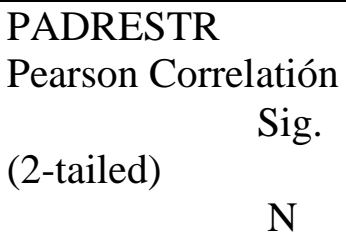 & $\begin{array}{l}.332 * * \\
.001 \\
92\end{array}$ & $\begin{array}{l}1.000 \\
92\end{array}$ \\
\hline
\end{tabular}

Entendiendo la escolaridad (asistencia o inasistencia a un centro educativo) en los niños mayores de tres años (61 sujetos) como un indicador de desarrollo adecuado y por ende de salud mental; tomamos esta variable y la correlacionamos con el tipo de clima familiar, para someter a contraste la segunda hipótesis específica. Los resultados presentados en la tabla 5 muestran el valor Chi-cuadrado 9.531, el cual es significativo en el nivel de 0.009 y con 2 grados de libertad. Lo que indica que ambas variables están asociadas. A su vez, la inspección de la tabla 6 señala que esta asociación es más fuerte en el tipo de clima familiar Balanceado, en el cual 20 niños están estudiando y solamente nueve no lo hacen; en tanto que por el contrario en la categoría de clima familiar Medio 17 sujetos no tienen escolaridad y seis si la tienen. En cambio entre las familias del tipo Extremo no hay mayor diferencia en cuanto a esta variable (cinco niños sin escolaridad y cuatro con escolaridad). 
Tabla $\mathbf{N}^{\circ} 5$

Ecuación de regresión (salud mental con estrés del padre y clima familiar

\begin{tabular}{|l|l|l|l|}
\cline { 2 - 4 } \multicolumn{1}{c|}{} & Value & df & $\begin{array}{c}\text { Asymp } \\
\text { Sig. } \\
\text { (2- } \\
\text { sided) }\end{array}$ \\
\hline $\begin{array}{l}\text { Pearson } \\
\text { Chi- Square } \\
\begin{array}{l}\text { N of valid } \\
\text { cases }\end{array}\end{array}$ & $9.531^{\mathrm{a}}$ & 2 & .009 \\
\hline
\end{tabular}

a. 2 cells $(33.3 \%)$ havwe expected count less than 5. the Minimum expected count is 4.43

Tabla $\mathbf{N}^{\circ} 6$

Contingencia: Escolaridad y clima familiar

\begin{tabular}{|c|c|c|c|c|c|}
\hline & \multicolumn{2}{|c|}{ Escol } & \multirow{2}{*}{ Total } \\
\hline & & & .00 & 1.00 & \\
\hline CFR & Extrema & $\begin{array}{l}\text { Count } \\
\text { Expected count } \\
\% \text { within CFR } \\
\% \text { within ESCOL } \\
\% \text { of total }\end{array}$ & $\begin{array}{c}5 \\
4.6 \\
55.6 \\
16.1 \\
8.2\end{array}$ & $\begin{array}{c}4 \\
4.4 \\
44.4 \\
13.3 \\
6.6\end{array}$ & $\begin{array}{c}9 \\
9.0 \\
100.0 \\
14.8 \\
14.8\end{array}$ \\
\hline & Media & $\begin{array}{l}\text { Count } \\
\text { Expected count } \\
\% \text { within CFR } \\
\% \text { within ESCOL } \\
\% \text { of total }\end{array}$ & $\begin{array}{c}17 \\
11.7 \\
73.9 \\
54.8 \\
27.9 \\
\end{array}$ & $\begin{array}{c}6 \\
11.3 \\
26.1 \\
20.0 \\
9.8 \\
\end{array}$ & $\begin{array}{c}23 \\
23.0 \\
100.0 \\
37.7 \\
37.7 \\
\end{array}$ \\
\hline & Balanceada & $\begin{array}{l}\text { Count } \\
\text { Expected count } \\
\text { \%within CFR } \\
\% \text { within ESCOL } \\
\% \text { of total }\end{array}$ & $\begin{array}{c}9 \\
14.7 \\
31.0 \\
29.0 \\
14.8\end{array}$ & $\begin{array}{c}20 \\
14.3 \\
69.0 \\
66.7 \\
32.8\end{array}$ & $\begin{array}{c}29 \\
29.0 \\
100.0 \\
47.5 \\
47.5\end{array}$ \\
\hline TOTAL & & $\begin{array}{l}\text { Count } \\
\text { Expected count } \\
\% \text { within CFR } \\
\% \text { within ESCOL } \\
\% \text { of total }\end{array}$ & $\begin{array}{c}31 \\
31.0 \\
50.8 \\
100.0 \\
50.8\end{array}$ & $\begin{array}{c}30 \\
30.0 \\
49.2 \\
100.0 \\
49.2\end{array}$ & $\begin{array}{c}61 \\
61.0 \\
100.0 \\
100.0 \\
100.0\end{array}$ \\
\hline
\end{tabular}

Para examinar en que medida las variables tipo de Clima Familiar y estrés del padre permiten predecir la Salud Mental del niño en la Tabla 7 se observan los resultados de contrastar un modelo que relacionaba estás tres variables.

En las Tablas No. 7a y 7b puede observarse el coeficiente de correlación múltiple (R) que alcanza un valor significativo de 0.332 , que señala una relación estadística significativa en la ecuación que tiene como variable dependiente la Salud Mental de los niños y como variables independientes las variables estrés del padre (EP) y tipo de clima familiar. 
Tabla No. 7

Ecuación de Regresión (salud mental con estrés del padre y clima familiar)

Tabla $\mathbf{N}^{\circ} 7 \mathbf{a}$

Resumen del modelo

\begin{tabular}{|c|c|c|c|c|}
\hline Model & R & $\begin{array}{c}\text { R. } \\
\text { Square }\end{array}$ & $\begin{array}{c}\text { Adjusted } \\
\text { R R. } \\
\text { Square }\end{array}$ & $\begin{array}{c}\text { Std Error } \\
\text { of the } \\
\text { estimate }\end{array}$ \\
\hline 1 & $.332^{\mathrm{a}}$ & .110 & .100 & 1.2205 \\
\hline
\end{tabular}

a. Predictors (constant), PADRESTR

Tabla $\mathbf{N}^{\circ} 7 \mathbf{b}$

Grado de sinificatividad estadística del modelo (ANOVA)

\begin{tabular}{|cc|l|c|l|c|c|}
\hline \multicolumn{1}{|c|}{ Model } & $\begin{array}{c}\text { Sum of } \\
\text { Squares }\end{array}$ & \multicolumn{1}{|c|}{ df } & $\begin{array}{c}\text { Mean } \\
\text { Square }\end{array}$ & \multicolumn{1}{|c|}{ F } & Sig. \\
\hline \multirow{2}{*}{1} & Regression & 16.595 & 1 & 16.595 & 11.141 & $.001^{\mathrm{a}}$ \\
& Residual & 134.057 & 90 & 1.490 & & \\
& Total & 150.652 & 92 & & & \\
\hline
\end{tabular}

a. Predictors (constant), PADRESTER

b. Dependent Variable: SM

La Tabla No. $7 \mathrm{~b}$ nos indica que el modelo ha quedado reducido a la variable independiente estrés del padre (EP) y la variable dependiente Salud Mental de los niños. La prueba $\mathrm{F}$ señala que este modelo así compuesto es estadísticamente muy significativo (nivel de significación 0.001)

Tabla $\mathbf{N}^{\circ} 7 \mathrm{c}$

Coeficientes Beta estandarizados y no estandarizados de la variable componente del modelo (Estrés del padre)

\begin{tabular}{|c|c|c|c|c|c|}
\hline \multirow{2}{*}{ Model } & \multicolumn{2}{|c|}{$\begin{array}{c}\text { Unstandarized } \\
\text { Coefficients }\end{array}$} & $\begin{array}{c}\text { Estándar. } \\
\text { Coefficien. }\end{array}$ & \multirow{2}{*}{ t } & \multirow{2}{*}{ Sig. } \\
\cline { 2 - 4 } & B & $\begin{array}{c}\text { Std. } \\
\text { Error }\end{array}$ & Beta & & \\
\hline 1 (constant) & .850 & .182 & & 4.678 & .000 \\
PADRESTR & $8.202 \mathrm{E}-02$ & 0.19 & .332 & 3.338 & .001 \\
\hline
\end{tabular}

a. Dependent Variable : SM

La Tabla No. 7c nos señala el coeficiente estandarizado de la variable predictora estrés del padre. Vale decir, nos señala «el peso» (0.332) con el cual interviene en la ecuación.

Finalmente, la Tabla No. 7d nos indica el peso de la variable tipo de Clima familiar que alcanza un valor $t$ no significativo $(\mathrm{t}<0.498)$; por lo cual fue excluida del modelo. 


\section{Tabla $\mathbf{N}^{\mathbf{0}} \mathbf{7 d}$}

Coeficiente Beta estandarizado de la variable excluida del modelo (clima familiar)

\begin{tabular}{|c|c|c|c|c|c|}
\hline Model & \multirow{2}{*}{ Beta In } & $\mathbf{t}$ & Sig. & $\begin{array}{c}\text { Partial } \\
\text { Correlation }\end{array}$ & $\begin{array}{c}\text { Collinearit y } \\
\text { Statistics }\end{array}$ \\
\cline { 4 - 6 } & & & & & \\
\hline 1. CLIMArance \\
\hline
\end{tabular}

a. Predictors in the model: (Constant) PSDRESTER

b. Dependent variable:

De otro lado ampliando el análisis resulta pertinente preguntarse en que medida los distintos tipos de clima familiar determinan diferentes grados de situaciones estresantes en los padres de estas familias.

\begin{tabular}{|c|c|c|c|c|c|c|}
\hline \multirow{3}{*}{ EPCOMPI } & \multicolumn{6}{|c|}{$\begin{array}{c}\text { Tabla } \mathbf{N}^{\circ} 8 \text { a } \\
\text { ANOVA: Estrés del padre y tipo de clima familiar } \\
\text { ANOVA }\end{array}$} \\
\hline & & $\begin{array}{c}\text { Sum of } \\
\text { Squares }\end{array}$ & df & $\begin{array}{c}\text { Mean } \\
\text { Square }\end{array}$ & $\mathbf{F}$ & Sig. \\
\hline & $\begin{array}{l}\text { Betwen Groups } \\
\text { Within Groups } \\
\text { Total }\end{array}$ & $\begin{array}{l}350.892 \\
3963.065 \\
4313.957\end{array}$ & $\begin{array}{l}2 \\
89 \\
91\end{array}$ & $\begin{array}{l}175.446 \\
44.529\end{array}$ & 3.940 & 0.23 \\
\hline
\end{tabular}

a. Dependet Varibale : SM

En la Tabla No. 8a se puede apreciar que la prueba F de ANOVA de una vía alcanza un valor estadísticamente significativo $(\mathrm{F}=3.94<0.023)$, este resultado nos indica que los tres grupos de la variable Clima Familiar difieren estadísticamente en sus promedios.

\section{Tabla $\mathbf{N}^{0} 8 b$ \\ Prueba Post Hoc LSD: Estrés del padre y tipo de clima familiar}

Dependent varibale : EPCOMPL

Multiple Comparisons

LSD

\begin{tabular}{|c|c|c|c|c|c|c|}
\hline \multirow{2}{*}{\multicolumn{2}{|c|}{ (I) CLIMAF (J)CLIMAF }} & \multirow{2}{*}{$\begin{array}{c}\text { Mean } \\
\text { Difference } \\
\text { (I-J) }\end{array}$} & \multirow[b]{2}{*}{$\begin{array}{l}\text { Stand. } \\
\text { Error }\end{array}$} & \multirow[b]{2}{*}{ Sig. } & \multicolumn{2}{|c|}{$\begin{array}{c}\text { 95\% Confidence } \\
\text { Interval }\end{array}$} \\
\hline & & & & & $\begin{array}{l}\text { Lower } \\
\text { Bound }\end{array}$ & $\begin{array}{l}\text { Upper } \\
\text { Bound }\end{array}$ \\
\hline \multirow[t]{2}{*}{1.0} & 2.00 & $3.4136^{*}$ & 1.4963 & .025 & .4404 & 6.3868 \\
\hline & 3.00 & -1.8004 & 2.1786 & .411 & -6.1292 & 2.5284 \\
\hline \multirow[t]{2}{*}{2.00} & 1.00 & $-3.4136^{*}$ & 1.4963 & .025 & -6.3868 & -.4404 \\
\hline & 3.00 & $-5.2140 *$ & 2.2168 & 0.21 & -9.6187 & -8.092 \\
\hline \multirow[t]{2}{*}{3.0} & 1.00 & 1.8004 & 2.1786 & .411 & -2.5284 & 6.1292 \\
\hline & 2.00 & $5.2140 *$ & 2.2168 & .021 & .8092 & 9.6187 \\
\hline
\end{tabular}

*. The mean diffenrence is significant at the .05 level

De otro lado en la Tabla No. 8b la prueba post hoc LSD nos permite precisar en que grupos de la variable Clima Familiar se dan las diferencias en relación a la variable estrés del Padre. En la tabla se observa que las diferencias se dan entre los tres grupos. La inspección de la columna Mean diference (i-J) indica que el grupo balanceado tiene un promedio más alto en estrés del padre que el grupo medio, y que a su vez el grupo extremo tiene también un promedio más elevado que el medio. De este modo se configura una agrupación en la variable estrés del padre: primer grupo compuesto por los grupos balanceado y extremo; segundo grupo, el grupo medio. 


\section{CONCLUSIONES}

Existe una relación significativa entre el número de acontecimientos estresantes experimentados por el padre de familia (o quien haga las veces del mismo) y el número de síntomas de problemas de salud mental que presentan sus niños.

Los niños de familias del tipo Medio, presentan significativamente menos escolaridad y dada la relación de esta variable con la salud mental es presumible que estos niños estarían en más riesgo de problemas de salud mental que los niños de familias del tipo Balanceado. Aunque los niños de las familias con estos dos tipos de clima familiar no presentan diferencias significativas en cuanto a su salud mental.

Existe una relación significativa entre el tipo de clima familiar y el número de acontecimientos estresantes experimentados por el padre de familia (o quien haga las veces del mismo); Las familias de los tipos "extremo y medio" tienen padres con un promedio de eventos estresantes signifícativamente más elevados que las familias de clima familiar tipo "balanceado".

\section{BIBLIOGRAFIA}

Bardales, Olga (1994) Relación entre autoestima, rendimiento académico y tipo de familia. Lima. Tesis. UPCH.

Benites, Luis (1998) Tipos de Familia, Clima social familiar y Asertividad en adolescentes. Lima. Tesis (Mg). USMP.

Boyden, J. (1988) Niños en situación de alto riesgo en Lima. Lima. UNICEF.

Castro, Jorge (1988) Salud mental e instrumento de registro en niños y adolescentes. Revista de Neuropsiquiatría, 38, 106-117.

Comisión especial del congreso (1998) Violencia en niños y adolescentes del Perú. Causas, consecuencias y recomendaciones.

Cuanto, (1997) La pobreza en el Perú de hoy. Midiendo la pobreza, Separata de información estadística.

Espinoza, Tula y Col. (1998) Estilos de vida y modelos de interacción familiar: su relación con problemas de salud mental. Lima. 1 Jornadas de Investigación en Salud. 63.

Feldman, S. (1985) Enfermedad mental y economía. Boletín Sanitario Panamericano, 98,201-213.

Lamas, Hector (1998) Violencia y persona. Psicología actual. Año VI, N 14-15. 5257.

Lasel, Friedrich (1994) La resilencia en el niño y en el adolescente. BICE. La infancia en el mundo. Vol. 5. 3. 8-11.

Livia, José y Ortiz, Mafalda (1993) Salud Mental Infantil en el Perú. Psicología actual. Año VI, $\mathrm{N}^{\circ} 14-15.45-51$.

Majluf. A. (1989) Prácticas de crianza en madres de status socio-económico medio y bajo de Lima. Revista de Psicología. 7. 151- 161.

Pimentel, Carmen (1988) Familia y violencia en la Barriada. Lima. TIPACON

Pimentel, Carmen (1988) Problemas psicológicos de los niños y represión Familiar y escolar en la Barriada . Lima.

Spielberger, Charles y Moscoso, Manolete (1996) Reacciones emocionales del estrés: Ansiedad y Cólera. Avances en Psicología Clínica Latinoamericana. V. 14. 59-81.

UNICEF (1997) Estado de la niñez, adolescencia y la mujer en el Perú. 